\title{
Wakefield Acceleration in Structures
}

\author{
Manoel E. Conde \\ Argonne National Laboratory \\ High Energy Physics Division \\ 9700 S. Cass Ave. Bldg. 362 \\ Argonne, IL 60439
}

\begin{abstract}
Wakefield acceleration in dielectric loaded structures is discussed in this paper. We present a description of the dielectric wakefield accelerator concept, comparing some features of the collinear and the two beam accelerator configurations. The Argonne Wakefield Accelerator Facility (AWA) is discussed in detail, including major upgrades that are presently taking place. The basic features and capabilities of the facility are presented, and the dielectric wakefield acceleration results are briefly summarized. Possible variants of the two beam accelerator configuration are discussed, and work on planar dielectric structures in various institutions is presented. We conclude this report mentioning prospective achievements of dielectric wakefield accelerating structures.
\end{abstract}

\section{INTRODUCTION}

The High Energy Physics accelerator community has no clear path to design linear colliders with energies higher than a few TeV. Therefore, new technology needs to be developed in order to make these higher energies accessible. The new technology needs to address the following three issues: (a) the energy source from which energy will be transferred to the beam; (b) the structure or medium that will support high accelerating gradients; (c) a means to transport and distribute the energy to the beam. We believe that electron beam driven accelerating schemes can make important contributions to these three issues.

Electron beam driven accelerators can be based on structures or plasmas. The latter is discussed in another paper in these proceedings [1]. This paper will report on the status of the development of dielectric based wakefield accelerators. Wakefield accelerators based on metallic structures (e.g. CLIC project at CERN [2]), and the use of active media for energy storage [3] will not be discussed here.

\section{DIELECTRIC WAKEFIELD ACCELERATOR CONCEPT}

Dielectric wakefield accelerators are dielectric loaded waveguides that are excited by the passage of electron beams. The wakefields generated by the "drive" bunches correspond to the Cherenkov radiation emitted by the electrons as they traverse the structures with a speed higher than the radiation group velocity supported by the structures. These devices are typically constructed as hollow cylindrical dielectric 
tubes inserted into cylindrical metallic waveguides, or as dielectric slabs attached to the walls of rectangular metallic waveguides. The simplicity of these devices is expected to make them relatively inexpensive. The absence of irises should allow operation at high accelerating gradients without electrical breakdown problems in the structures. These dielectric structures compare favorably with conventional iris loaded structures in terms of damping of higher order modes [4].

Figure 1 shows the longitudinal cross section of a cylindrical dielectric loaded accelerator. The intensity of the accelerating field $W_{z}$ generated by the passage of an electron bunch of charge $Q$ and bunch length $\sigma_{z}$ is given approximately by

$$
W_{Z} \approx \frac{Q}{a^{3 / 2}} \exp \left[-2\left(\frac{\pi \sigma_{z}}{\lambda_{n}}\right)^{2}\right]
$$

where $a$ is the inner radius of the dielectric tube, and $\lambda_{n}$ is the wavelength of the $n$-th mode supported by the structure. The expression shows that it is desirable to have high charge bunches traversing small diameter structures. An example of a dielectric structure, including the beam parameters and resulting wakefield is given in Table 1.

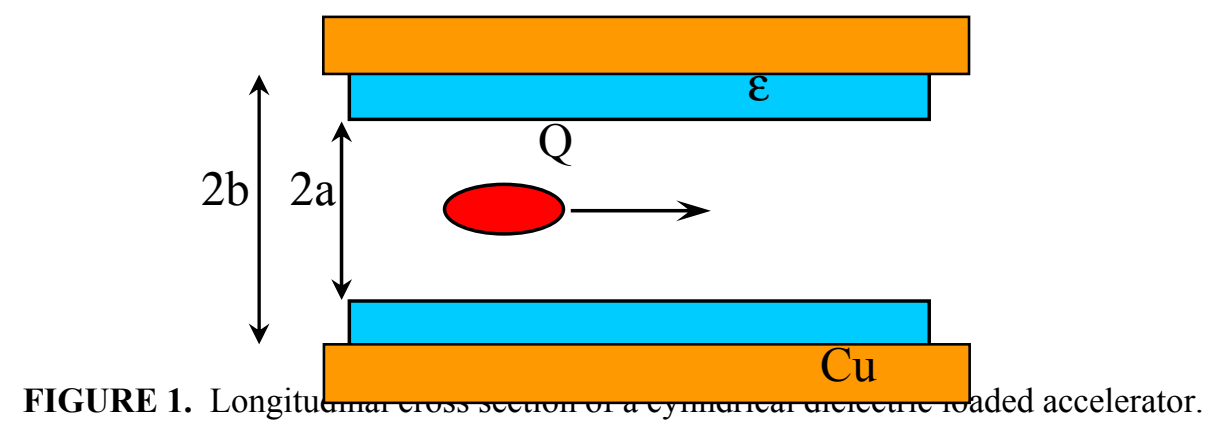

TABLE 1. Example of cylindrical dielectric loaded structure.

Inner diameter of dielectric $(2 a)$

Outer diameter of dielectric $(2 b)$

$6 \mathrm{~mm}$

Dielectric constant $(\varepsilon)$

$10 \mathrm{~mm}$

Charge of electron bunch $(Q)$

Bunch length $\left(\sigma_{z}\right)$

RF power generated $(P)$

$00 \mathrm{nC}$

$\mathrm{RF}$ frequency $(f)$

$1 \mathrm{~mm}$

Accelerating gradient $\left(W_{z}\right)$

$400 \mathrm{MW}$

$19 \mathrm{GHz}$

$92 \mathrm{MV} / \mathrm{m}$

A bunch train of four $100 \mathrm{nC}$ bunches or eight $50 \mathrm{nC}$ bunches traversing the device described in Table 1 would generate an accelerating gradient of over $300 \mathrm{MV} / \mathrm{m}$.

Dielectric wakefield accelerators can be operated in the collinear configuration, where the same structure is used to decelerate the drive bunches and to accelerate the probe bunches. That is, an electron bunch traverses the dielectric loaded structure, emitting radiation and establishing the wakefield in the structure; this drive bunch is followed by another electron bunch (probe or witness bunch) which is accelerated by the wakefield present in the structure. 
Alternatively, dielectric wakefield accelerators can be operated in the two beam accelerator configuration (TBA), where two dielectric loaded structures are used (Fig. 2 ). The RF power generated by the drive beam is coupled out of the decelerating structure and into a second dielectric loaded structure, where the witness beam is accelerated.

\section{Schematic Diagram of Argonne Wakefield Step-Up Transformer}
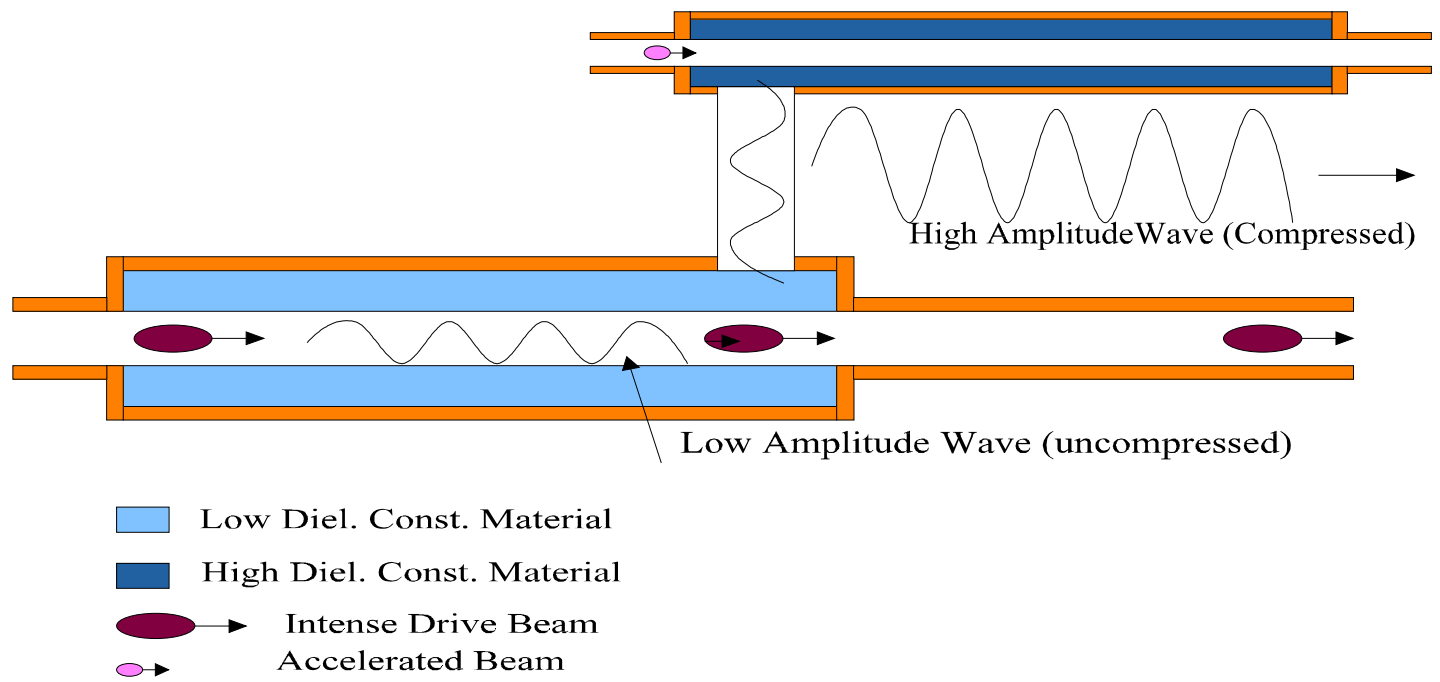

FIGURE 2. Schematic of a dielectric wakefield two beam accelerator, also known as step-up transformer.

The two beam accelerator scheme offers several advantages over the collinear mode of operation. In the collinear configuration, the so-called transformer ratio cannot have a value greater than two if the longitudinal distribution of charge is symmetric with respect to the center of the bunch; that is, for symmetric bunches the rate at which the witness beam gains energy cannot exceed twice the rate at which the drive beam loses energy. This limitation is easily avoided in the two beam accelerator scheme, where the RF pulse can be compressed in the second structure, both in the transverse plane (essentially by having the second structure with smaller dimensions) and in the longitudinal dimension (by using a higher dielectric constant material in the second structure, with the corresponding lower group velocity for the radiation). The possibility of RF pulse compression, with the consequent higher accelerating gradient in the second structure, is the reason why this configuration is also known as "step-up transformer".

Both configurations can make use of multiple drive bunches, in the form of a bunch train, allowing for the build up of the fields inside the structure. In this way, both schemes can achieve higher accelerating gradients; the collinear case, however, is still subject to the transformer ratio limit of two.

Both schemes can make use of multiple stages of acceleration (and deceleration) to reach higher final energies. This would require several drive bunches (or several drive bunch trains) to traverse several structures. While this is possible in the collinear 
configuration, it is certainly simpler in the two beam accelerator case, because of the greater flexibility in terms of the beam optics.

\section{ARGONNE WAKEFIELD ACCELERATOR FACILITY (AWA)}

The Argonne Wakefield Accelerator Facility (AWA) was designed to study wakefield acceleration. The facility uses a high charge drive beam to excite wakefields, and a low charge witness beam to probe the wakefields. These two beams can be made to propagate through the same structure, to study collinear acceleration, or can travel to two separate structures (Fig. 3).

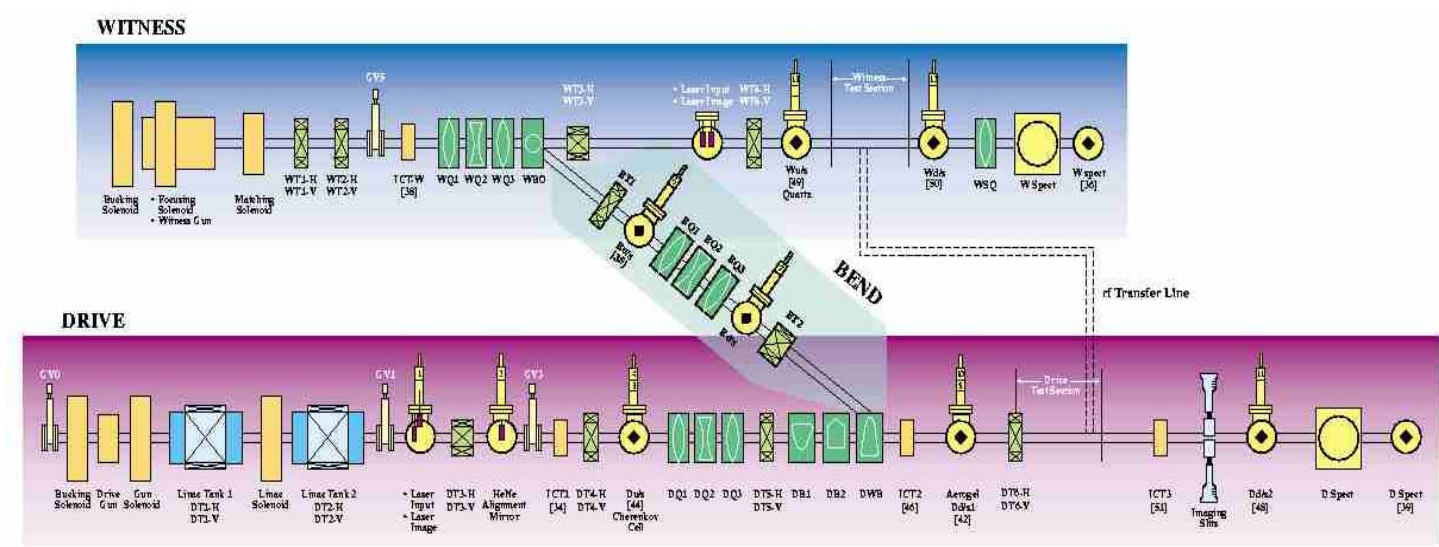

FIGURE 3. Schematic of the Argonne Wakefield Accelerator beamlines.

The drive beam is generated by a half-cell photocathode RF gun running at 1.3 $\mathrm{GHz}$. The bunch charge can be varied from 10 to $100 \mathrm{nC}$, with a bunch length of 15 to 35 ps FWHM. The drive gun is followed by two linac tanks $(1.3 \mathrm{GHz})$ that bring the beam energy up from $2 \mathrm{MeV}$ to $15 \mathrm{MeV}$.

The $4 \mathrm{MeV}$ witness beam is generated by a $6 \frac{1}{2}$ cell photocathode RF gun. The bunch charge is typically $0.1 \mathrm{nC}$ with a bunch length of 8 ps FWHM. Both guns, as well as the linac tanks, are powered by a single $30 \mathrm{MW}$ L-band klystron.

The laser system consists of a dye oscillator $(496 \mathrm{~nm})$ followed by a dye amplifier and an excimer amplifier; a doubling crystal is followed by a second excimer amplifier (248 nm), bringing the final pulse energy to $8 \mathrm{~mJ}$, with a pulse duration of 6 to $8 \mathrm{ps}$ FWHM.

Over the past few years, several experiments have been successfully carried out at the AWA facility in both the collinear and the two beam accelerator configurations [5]. All these experiments used dielectric loaded cylindrical waveguides, with operating frequencies ranging from 7 to $20 \mathrm{GHz}$. The dielectric materials are typically Magnesium-Calcium-Titanate based ceramics (MCT), with dielectric constants spanning from 4 to 40 . Accelerating fields of about $15 \mathrm{MV} / \mathrm{m}$ have been measured in collinear acceleration experiments. A two beam accelerator experiment operating at $7.8 \mathrm{GHz}$ demonstrated that more than $90 \%$ of the RF power generated by the drive 
beam (4 MW) was coupled into the second structure, yielding an accelerating field of $7 \mathrm{MV} / \mathrm{m}$. Figure 4 shows the energy modulation of the witness beam as it enters the dielectric loaded structure at different values of the RF phase.

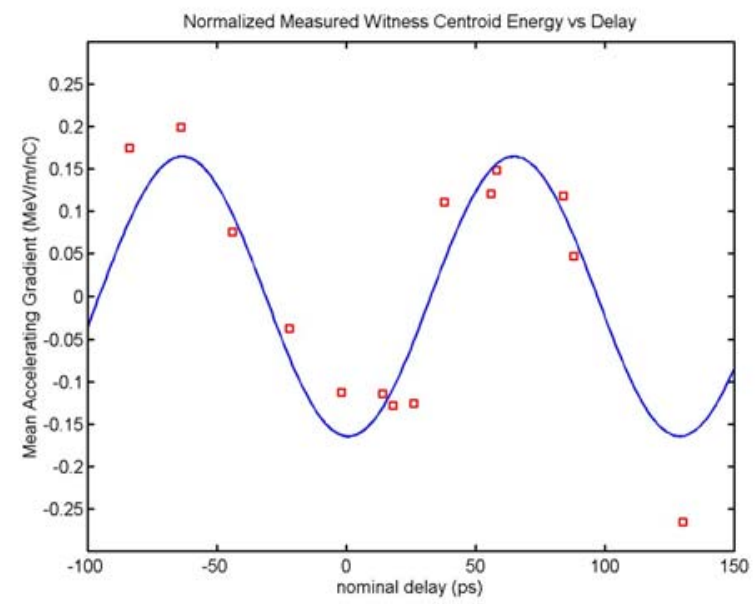

FIGURE 4. Plot showing the witness beam energy as a function the delay beam the witness and the drive beams.

Plasma wakefield acceleration experiments have also been carried out at the AWA facility. Electron beam focusing and acceleration in the underdense (blowout) regime were first observed with the AWA beam [6].

Much higher accelerating gradients can be achieved if the quality of the drive beam increases. Smaller emittances allow the operation of smaller diameter structures, which yield much higher accelerating fields. Shorter electron bunches would also permit operation at higher RF frequencies. The field superposition resulting from the operation with longer bunch trains would allow the RF fields to build up to higher levels as well. For these reasons the AWA facility is currently undergoing several major upgrades.

A new photocathode RF gun has been built to replace the old drive beam gun. The old gun had been designed and built when only 2 MW of RF power was available. This limited amount of RF power had led to the construction of a half-cell gun, yielding a relatively soft $2 \mathrm{MeV}$ electron beam at the gun exit, with the consequent high emittance and long bunch length. The new $1 \frac{1}{2}$ cell gun is expected to produce a $7.5 \mathrm{MeV}$ beam with $12 \mathrm{MW}$ of RF power. Similar bunch charges $(10-100 \mathrm{nC})$ will be generated by the new gun, but with shorter bunch lengths $(2-5 \mathrm{ps}$ rms $)$ and much lower emittances $(30-200 \pi \mathrm{mm}$ mrad). The new gun (Fig. 5) has been conditioned up to $12 \mathrm{MW}$ of power, and produced the first photoelectron beam during the last few hours of operation of the old laser system. The beam will be fully diagnosed once operation with the new laser system starts.

Installation of the new laser system was completed a few days before the start of this workshop. The new system consists of a Spectra Physics Tsunami oscillator followed by a Spitfire regenerative amplifier and two Ti:Sapphire amplifiers (TSA 50). It produces $1.5 \mathrm{~mJ}$ pulses at $248 \mathrm{~nm}$, with a pulse length of 6 to $8 \mathrm{ps}$ FWHM and a repetition rate of up to 10 pps. The very brief experience we have had running this 
new system confirms that its power stability and beam profile quality are indeed much better than what the old laser system could provide.

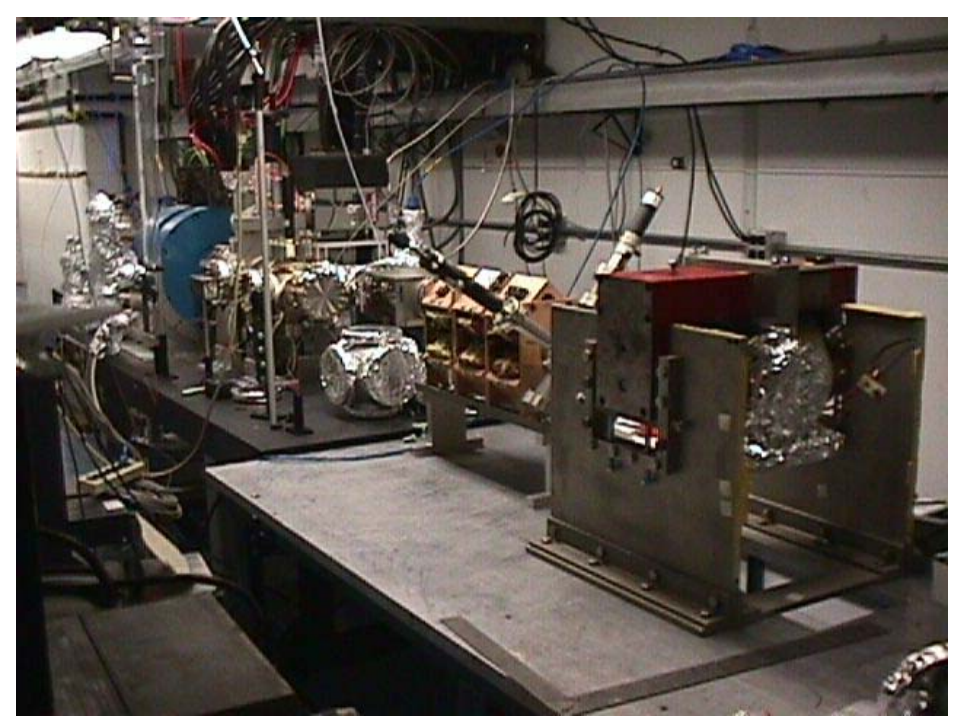

FIGURE 5. Picture of the new AWA drive gun under installation.

The third item being upgraded is the photocathode material. The old drive gun operated routinely with magnesium photocathodes, being able to produce up to $100 \mathrm{nC}$ of charge with $5 \mathrm{~mJ}$ of UV laser beam (quantum efficiency of $1 \times 10^{-4}$ ). The new drive beam has been commissioned with a copper photocathode, but that will soon be replaced by a cesium telluride photocathode [7], which is expected to have a quantum efficiency close to $10 \%$. We need a quantum efficiency of about $1 \%$ in order to generate 64 bunches of $50 \mathrm{nC}$ with $1.5 \mathrm{~mJ}$ of laser energy at $248 \mathrm{~nm}$.

\section{VARIANTS OF THE TWO BEAM ACCELERATOR SCHEME}

There are two possible variants of the dielectric two beam accelerator scheme: (a) the structure where the drive beam loses energy can be used as a power source for metallic accelerating structures; (b) a dielectric loaded structure can be powered by a conventional RF source.

Both of these options are currently being pursued by the AWA group in collaboration with other research institutions. Duly Research, in collaboration with CERN and ANL, is building $21 \mathrm{GHz}$ dielectric loaded structures to be tested as power sources using the CTF II electron beam at CERN as the drive beam [8]. These structures are expected to generate more than $150 \mathrm{MW}$ of RF power at $21 \mathrm{GHz}$.

The AWA group has built $11.4 \mathrm{GHz}$ dielectric loaded prototype structures to be tested at NRL using their X-band magnicon as the RF power source [9]. 


\section{PLANAR DIELECTRIC WAKEFIELD STRUCTURES}

W-band dielectric loaded structures have been built and tested using the NLCTA electron beam at SLAC [10]. These planar dielectric structures were constructed by inserting two alumina slabs into a rectangular waveguide section. The transverse dimensions of the structures are of the order of hundreds of micrometers, with a 720 $\mu \mathrm{m}$ gap for the passage of the $300 \mathrm{MeV}$ electron beam. One of these devices was configured in a ring resonator circuit, where the measurements indicated that $200 \mathrm{~kW}$ of circulating power generated an accelerating field of $20 \mathrm{MV} / \mathrm{m}$.

A collaboration between Yale, Columbia and Omega-P is developing planar dielectric loaded structures with transverse dimensions of the order of tens of micrometers. They plan to chop the electron beam from the accelerator LACARA [11], in order to make $1 \mu \mathrm{m}$ long bunches of $1 \mathrm{pC}$ charge. A bunch train composed of ten of these small bunches, each spaced by $20 \mu \mathrm{m}$, is expected to generate an accelerating field of about $600 \mathrm{MV} / \mathrm{m}$.

\section{CONCLUSION}

Substantial progress is being made in the development of dielectric loaded structures as an alternative to the use of metallic structures. The AWA upgrades will make it a powerful tool for the study of electron beam driven accelerators and the exploration of higher accelerating gradients. The new AWA beam will be capable of demonstrating gradients of the order of 200 to $300 \mathrm{MV} / \mathrm{m}$ in dielectrics, in both collinear and step-up transformer structures. This intense beam will be able to generate hundreds of megawatts of RF power with frequencies in the range of 30 to $100 \mathrm{GHz}$, with pulse lengths of tens of nanoseconds, assuring a prominent place for beam driven schemes in the quest for multi TeV colliders.

\section{ACKNOWLEDGMENTS}

This work was supported by DOE, High Energy Physics Division, Advanced Technology Branch, under Contract No. W-31-109-ENG-38.

\section{REFERENCES}

1. Barov, N., "Plasma-Wakefield Acceleration Experiments", in these proceedings.

2. Corsini, R., "An Overview of the New CLIC Test Facility (CTF3)" in Particle Accelerator Conference-2001, edited by P. Lucas and S. Webber, Chicago, 2001, pp. 412-414.

3. Schachter, L., Colby, E., and Siemann, R. H., "Wake-Amplification by a Solid-State Active Medium" in Advanced Accelerator Concepts Workshop-2000, edited by P. L. Colestock and S. Kelley, AIP Conference Proceedings 569, New York: American Institute of Physics, 2001, pp. 863872.

4. Chojnacki, E. et al., J. Appl. Phys. 69, 6257 (1991).

5. Gai, W. et al., "Experimental Demonstration of Two Beam Acceleration Using Dielectric Step-Up Transformer" in Particle Accelerator Conference-2001, edited by P. Lucas and S. Webber, Chicago, 2001, pp. 1880-1882. 
6. Barov, N. et al., Phys. Rev. ST Accel. Beams 3, 011301 (2000).

7. Nguyen, D., private communication.

8. Yu, D., in these proceedings.

9. Power, J. G. et al., "High Power Testing of ANL X-Band Dielectric-Loaded Accelerating Structures" in these proceedings.

10. Hill, M. E. et al., Phys. Rev. Letters 87, 094801 (2001).

11. Hirshfield, J. L., and Wang, C., "Laser-Driven Cyclotron Autoresonance Accelerator" in Advanced Accelerator Concepts Workshop-2000, edited by P. L. Colestock and S. Kelley, AIP Conference Proceedings 569, New York: American Institute of Physics, 2001, pp. 326-334. 